\title{
Profound hypertension with dexmedetomidine during insertion of deep brain stimulator
}

\author{
Allison Tedder, Evgeny Rakhman, Pirjo Manninen, Lashmikumar Venkatraghavan
}

\begin{abstract}
Dexmedetomidine is now frequently used in the anaesthetic management of patients undergoing deep brain stimulator insertion for movement disorders. We present two patients with Parkinson's disease and dystonia who developed marked increase in blood pressure and level of sedation during the infusion of a loading dose of dexmedetomidine $(1 \mathrm{mcg} / \mathrm{kg}$ over $10 \mathrm{~min})$. Both patients required treatment of their blood pressure. The first patient also had a computed tomography of the brain to rule out an intracranial event. The patients recovered from these untoward events in approximately $30 \mathrm{~min}$. The possible explanations for both the hypertension and oversedation were underestimation of the severity of the patients' underlying disease process and a relative overdose of the loading dose of dexmedetomidine.
\end{abstract}

Key words: Deep brain stimulation, dexmedetomidine, hypertension, Parkinson's disease

\section{INTRODUCTION}

The insertion of deep brain stimulators (DBS) is now common for the treatment of movement disorders such as dystonia and Parkinson's disease. ${ }^{[1,2]}$ The anaesthetic management of the patient includes the use of monitored anaesthesia care with and without sedation and general anaesthesia. Dexmedetomidine an alpha adrenoreceptor agonist is gaining popularity for use in these procedures. ${ }^{[1-4]}$ The advantages of dexmedetomidine are that it provides anxiolysis, sedation with minimal risk of respiratory depression, and hemodynamic instability though changes in blood pressure, both hypotension and hypertension, have been

\begin{tabular}{|l|l|}
\hline \multicolumn{2}{|c|}{ Access this article online } \\
\hline Quick Response Code: & Website: \\
\hline & www.jnaccjournal.org \\
\cline { 2 - 2 } & \\
\hline
\end{tabular}

noted to occur. ${ }^{[4-6]}$ We presented two cases of severe hypertension and marked oversedation during the loading dose infusion of dexmedetomidine sedation in patients undergoing DBS insertion. Written informed consent was obtained from both patients.

\section{CASE REPORTS}

\section{Case 1}

A 70-year-old, $70 \mathrm{~kg}$ man, American Society of Anesthesiologist (ASA) 3, with severe orofacial dystonia presented for a bilateral insertion of DBS into the globus pallidus interna (GPI) nucleus. Medical history was significant for hypertension, mild obstructive lung disease, and past history of myasthenia gravis treated with a thymectomy. Current medications included tetrabenazine, clonazepam, ramipril, azathioprine, and advair. During his preoperative assessment, blood pressure was $115 / 77 \mathrm{mmHg}$, heart rate $77 \mathrm{bpm}$ and his electrocardiogram (ECG) was normal. Preoperatively the patient took all his routine medications. On the morning of surgery in the magnetic resonance imaging suite, a stereotactic Leksell head frame was placed

Department of Anesthesia, University of Toronto, Toronto Western Hospital, University Health Network, Toronto, Ontario, Canada

Address for correspondence:

Dr. Lakshmikumar Venkatraghavan, Department of Anesthesia, Toronto Western Hospital, 399, Bathurst Street, Toronto, Ontario M5T 2S8, Canada. E-mail: Lashmi.Venkatraghavan@uhn.ca 
with infiltration of local anaesthesia (lidocaine $1 \%$ with epinephrine $1: 200,000$ ) by the surgeon but with no sedation, followed by imaging to target the GPI. Patient was then brought to the operating room and placed in a semi-sitting position on the operating room table with attachment of the Leskell head frame to the table. Standard monitors were used included ECG, noninvasive blood pressure, pulse oximetre, and end-tidal $\mathrm{CO}_{2}$ via nasal probes used for delivery of supplemental oxygen $(4 \mathrm{l} / \mathrm{min})$. After establishing baseline vital signs (blood pressure $150 / 80 \mathrm{mmHg}$, heart rate $60 \mathrm{bpm}$ ), a loading dose of intravenous (IV) dexmedetomidine at $1 \mathrm{mcg} / \mathrm{kg}$ (70 mcg) was administered over $10 \mathrm{~min}$ period. During the procedure, the intraoperative sedation/alertness was assessed using the Modified Observer's Assessment of Alertness/Sedation Scale with aim to maintain the level of sedation score of $3 .^{[5]}$ The only other medication administered was an antibiotic (cephazolin $2 \mathrm{~g}$ ). Toward the end of the 10 min infusion of dexmedetomidine, infiltration the scalp with local anaesthetic (lidocaine 1\% with epinephrine 1:200,000) for the placement of burr holes was started by the surgeon. However, at this time it was noted that the patient was severely obtunded and not responding to commands or stimulation. The blood pressure had increased to $230 / 100 \mathrm{mmHg}$ with a pulse of $65 \mathrm{bpm}$. Respiratory rate was 14 breaths/min and oxygen saturation $\left(\mathrm{SaO}_{2}\right)$ was $98 \%$. Pupil size was small bilaterally. Dexmedetomidine infusion was stopped immediately. Labetolol $10 \mathrm{mg}$ IV bolus was administered, but blood pressure remained above 180/90 $\mathrm{mmHg}$ with a heart rate of $62 \mathrm{bpm}$. After discussion with the neurosurgeons, the patient was transferred for immediate computerized tomography (CT) scan of the brain. This was found to be negative. Surgery was cancelled, and he was transferred to the postanaesthetic care unit (PACU). On arrival in the PACU his blood pressure was $120 / 80 \mathrm{mmHg}$, pulse $60 \mathrm{bpm}, \mathrm{SaO}_{2} 98 \%$, and he was alert and orientated with no change in his neurological status. Patient was observed in the PACU for $2 \mathrm{~h}$ and then overnight in the neurosurgical step down unit with no untoward events. He was discharged from the hospital on the second postoperative day. Two weeks later, he underwent the same planned procedure with conscious sedation using remifentanil and propofol infusions without any incident. He had a good outcome.

\section{Case 2}

A 70-year-old, $105 \mathrm{~kg}$ man, ASA 3 was scheduled for insertion of bilateral DBS at GPI for Parkinson's disease. His medical history included hypertension and prostate hypertrophy, and ongoing medications were nadolol, coversyl, sinemet, amantadine, and tamsulosin. This patient was managed in a similar fashion to Case 1. After positioning in the operating room a dexmedetomidine loading dose infusion $1 \mathrm{mcg} / \mathrm{kg}$ over $10 \mathrm{~min}(100 \mathrm{mcg})$ was initiated. Towards the end of the loading dose the patient became deeply sedated and disoriented. Blood pressure increased to $220 / 95 \mathrm{mmHg}$ from his initial reading of $150 / 80 \mathrm{mmHg}$ and heart rate decreased to 50 $\mathrm{bpm}$ from $80 \mathrm{bpm}$. The dexmedetomidine infusion was stopped, and the patient was treated by IV boluses of a total amount of hydralazine $20 \mathrm{mg}$ and nitroglycerine $100 \mathrm{mcg}$. The patient's blood pressure and heart rate and level of consciousness gradually returned to the initial parameters over approximately $10 \mathrm{~min}$. No further sedation was administered. After discussion with the surgeons, the surgery was started and the procedure was completed without any other further incidents. The patient had a good outcome.

\section{DISCUSSION}

We present the occurrence of the profound hypertension and over sedation in two patients during the infusion of a loading dose $(1 \mathrm{mcg} / \mathrm{kg}$ over $10 \mathrm{~min})$ of dexmedetomidine. In both patients, this occurred towards the end of the loading dose. The patients were heavily sedated and unable to respond to questions and commands and were noted to have a marked increase in blood pressure. The exaggerated responses were unexpected. The marked hypertension could have been related to a preoperative history of hypertension with addition of anxiety and possibly discomfort during the positioning of the patient or injection of local anaesthesia, or even due to the presence of epinephrine in the local anaesthetic. However, both patients were also very sedated, which could not be explained by this. Due to the unresponsiveness of the first patient, there was a concern that he may have developed an intracranial event. Thus, a CT scan was performed. The possible reasons for these events include the administration of a relative overdose. The first patient had a history of myasthenia gravis, and his medical condition may have been underestimated. The second patient received a dose calculated on total body weight $(100 \mathrm{~kg})$ which in view of his Parkinson's disease may have been too high. Parkinson's disease is a multisystem disorder with varying degree of symptoms, thus some patients may be more sensitive to various medications especially sedative agents.

Dexmedetomidine is alpha2-adrenoreceptor agonist with a differential specificity for the alpha2 receptors. ${ }^{[4,6]}$ Dexmedetomidine produces sedation with patients calm, comfortable, yet alert enough to respond and to cooperate in obeying commands. This sedative effect is centrally mediated through alpha2A receptors located in the locus ceruleus, which may allow cortical neurons to continue functioning upon stimulation in comparison to other sedative agents such as propofol and barbiturates. ${ }^{[4]}$ Thus, dexmedetomidine sedation is considered ideal when intraoperative neurophysiologic 
monitoring in an awake patient is required as it does not directly affect the activity of subthalamic neurons. Rozet et al. showed that dexmedetomidine did not impair the intensity of movement disorders in Parkinson's disease nor did it interfere with the microelectrode recordings. ${ }^{[3]}$

Other properties that make dexmedetomidine an ideal agent during DBS surgery is that it maintains hemodynamic stability and causes minimal respiratory depression. However, there are other side-effects, which include hypotension, hypertension, bradycardia, worsening of heart block, dry mouth, and nausea. ${ }^{[6]}$ The manufacturer recommendations are to administer the loading dose over $10 \mathrm{~min}$ to minimize these side-effects. The effects of dexmedetomidine on blood pressure are biphasic with an initial transient rise with a reflex fall in heart rate brought about by stimulation of alpha2B subtypes of receptors present in vascular smooth muscles. ${ }^{[7,8]}$ This is followed by fall in blood pressure and heart rate due to inhibition of central sympathetic outflow and stimulation of presynaptic alpha2 receptors resulting in a decreased release of nor-adrenaline leading to further fall in the blood pressure. The hypertension associated with dexmedetomidine is usually mild, transient and not requiring treatment. This was not the case in our patients.

The pharmacokinetic properties of dexmedetomidine show a rapid distribution phase with a distribution half-life of about $6 \mathrm{~min}$ and a terminal elimination half-life of $2 \mathrm{~h} \cdot{ }^{[6,7]}$ As well metabolism of dexmedetomidine is not affected by gender or age in patients older than 18 years. Both of our patients recovered from the adverse effects (hypertension and over sedation) of the drug within $30 \mathrm{~min}$.

Our recommendations in the use of dexmedetomidine in patients with functional disorders especially Parkinson's disease and dystonia for DBS insertion is to decrease the total loading dose and/or increase the duration of the administration of a loading dose. As well one should consider the use of an ideal body weight for calculation of the dose.

\section{REFERENCES}

1. Rozet I. Anesthesia for functional neurosurgery: The role of dexmedetomidine. Curr Opin Anaesthesiol 2008;21:537-43.

2. Bekker A, Sturaitis MK. Dexmedetomidine for neurological surgery. Neurosurgery 2005;57:1-10.

3. Rozet I, Muangman S, Vavilala MS, Lee LA, Souter MJ, Domino $\mathrm{KJ}$, et al. Clinical experience with dexmedetomidine for implantation of deep brain stimulators in Parkinson's disease. Anesth Analg 2006;103:1224-8.

4. Maurtua MA, Cata JP, Martirena M, Deogaonkar M, Rezai A, Sung W, et al. Dexmedetomidine for deep brain stimulator placement in a child with primary generalized dystonia: Case report and literature review. J Clin Anesth 2009;21:213-6.

5. Chernik DA, Gillings D, Laine H, Hendler J, Silver JM, Davidson $\mathrm{AB}$, et al. Validity and reliability of the Observer's Assessment of Alertness/Sedation Scale: Study with intravenous midazolam. J Clin Psychopharmacol 1990;10:244-51.

6. Bajwa S, Kulshrestha A. Dexmedetomidine: An adjuvant making large inroads into clinical practice. Ann Med Health Sci Res 2013;3:475-83.

7. Bloor BC,Ward DS, Belleville JP, Maze M.Effects ofintravenous dexmedetomidine in humans. II. Hemodynamic changes. Anesthesiology 1992;77:1134-42.

8. Dyck JB, Maze M, Haack C, Vuorilehto L, Shafer SL. The pharmacokinetics and hemodynamic effects of intravenous and intramuscular dexmedetomidine hydrochloride in adult human volunteers. Anesthesiology 1993;78:813-20.

How to cite this article: Tedder A, Rakhman E, Manninen $\mathrm{P}$, Venkatraghavan L. Profound hypertension with dexmedetomidine during insertion of deep brain stimulator. J Neuroanaesthesiol Crit Care 2015;2:55-7.

Source of Support: Nil, Conflict of Interest: None declared. 